\title{
Research on Hospital Intelligent Monitoring System Based on Internet of Things
}

\section{ZHOU Huachun}

${ }^{1}$ Department of the internet of things ,Chongqing College of electronic engineering, ChongQing, 401331, China

a420749294@qq.com

Keywords: Hospital Intelligent Monitoring System, Internet of Things

\begin{abstract}
Health services are in times with rapid development and ever-changing. With the treatment of an aging population structure, disease prevention and control is gradually transformed into the new medical model, which requires a new generation of digital sound engineering technology to digital, intelligent direction. Things technology in the hospital network through ubiquitous sensing device interoperability, support health care information collection automation, intelligent transport, a comprehensive collection of global decision-making and analysis of the whole process of assistance, to improve the capacity and efficiency of medical services, improve the quality of medical services and model to achieve full health-oriented management of medical wisdom.
\end{abstract}

\section{Introduction}

Things with major information technology development and progress, is regarded as a major opportunity and development opportunities in today's field of information processing and network technology development, the development of networking technology in the domestic rise in 2009. The concept, thus the rise of the Internet of Things technology development boom, things are composed of a network layer, the perception layer and application layer, network layer which is the key and core technology development, but also to promote the fundamental networking technology. Things of this concept and key technologies include support for all aspects of modern signal processing and network technology, such as sensing and detection technology, identification and recognition technology, signal processing technology, computer networks and communication technologies and information security technologies.

\section{The Concept of the Internet of Things}

RFID (radio frequency identification) technology is a wireless automatic identification technology, also known as RFID technology, RFID technology has many advantages, widely used in transportation, logistics, safety, security, medical, etc., as a bar code identification technology upgrading products in recent years, a huge market demand makes RFID has developed rapidly.

Things connotation is originated by the RFID objective objects represented using the network to exchange data and this concept, and continue to expand, extend, and improve gradually formed. That emphasizes terminals and processing is defined by radio frequency identification, infrared sensors, global positioning systems, laser scanners and other information sensing device, according to the agreed protocol, to any article connected to the Internet, information exchange and communication, in order to achieve intelligent identify, locate, track, monitor and manage a network technology called Internet of things technology.

Things break with the current exchange all Internet applications characterized by boundary will extend the network to the application objects and objects, machines and machine, between the material and the machine, and objects, man and machine, the industry needs, network, computers, electronics, information technology and management methods developed to a certain stage of the inevitable product. 


\section{Demand Analysis}

Daily demand must be measured according to the general ward body temperature, pulse and blood pressure three parameters, analysis of engineering processes and data collection in the existing problems, to find the best solution. Current hospital ward nursing work processes cumbersome, inefficient, and the potential risks, nurses day of the patient's temperature and pulse several times (according to the patient's condition to choose between 1-6 times) measurements. Currently measured temperature process is as follows: First, the ward by a nurse under a mercury thermometer given to each patient was measured after 10 minutes and the nurse at the recovery ward again, read the temperature data in the recovery process and recorded on a single temperature. Measuring pulse and blood pressure is as follows: measure the patient by a nurse at the ward wrist pulse with your fingers, using a sphygmomanometer blood pressure measurement, and then recorded on paper records. Above work every day nurses occupy a lot of work time and increases the hospital's operating costs. In a large three hospitals, for example, in the hospital from 2008 to 2010, the number of annual consumption of mercury thermometer showed an increasing trend, so the loss of mercury per year is about 20KG. After the mercury leak after people tend to collect particles of mercury, into the trash or sewer, this approach would lead to serious soil pollution and groundwater resources. To reduce the impact on the environment, a common practice is to clean up mercury with sulfur powder particles, but the particles are more scattered, and some particles are too small, cannot clean up the residual mercury particles will evaporate quickly at room temperature, resulting in mercury Pollution. If mercury thermometer mercury completely evaporated, can make an area of 15 square meters, 3 meters high room indoor air mercury exceeded 800 times.

Based on the above analysis of the demand, an urgent need to design performance wireless networking technology based on intelligence collected daily average patient needs care system acquisition parameters to improve the efficiency of care, reduce medical errors, and to address the clinical problem of mercury pollution.

\section{The Construction of Guardianship Framework based on the Internet of Things}

Using networking technology to build a network monitoring system, including background monitoring center, network and user side of things is guardianship, various types of detector. Critical patient monitoring network system architecture usually three things the system, as described above, namely, architecture perception layer, network layer and application layer.

The Perception Layer. Perception layer directly underlying object-oriented side, the main task is to collect specific information embodied glucose monitors, blood pressure monitors, heart monitors and other patient lives, the underlying medical device monitoring network monitoring system.

The Network Layer. The network layer is the key to this system of things set up, including the Internet, GPRS, 3G, Wifi networks, set up by networking, and information sharing and multi-network transport network combined.

The Application Layer. Application layer is the top system in this system is mainly reflected in the smart health care system and application management, while taking advantage of the decision-making robot technology, to monitor the information processing, decision-making and monitoring values are judged to tell whether the patient is disease and give some corresponding solutions. For example, intelligent remote monitoring of blood glucose, blood pressure monitoring, cardiac function monitoring, self-health management, emergency rescue, etc., to achieve the health sector in charge of the patient-friendly care.

\section{The System Design}

According to clinical care needs in conjunction with the system application environment, system design must address the following issues when: (1) the portable device. The sensor is concentrated in high reliability of a portable device to meet the small size, easy to use requirements, and has high 
accuracy performance. (2) low power consumption. Since the sensing terminal device more frequently used, but the small amount of data collected, and does not require a high transmission rate, and therefore, low-power device can ensure a long time, without the need for frequent recharging. (3) network automatically. Each ward has 40 beds at least, so the need to allot at least 40 sets of senses that the terminal equipment. This is composed of 40 sets of equipment for an automatic network and transmits vital signs parameters wirelessly to the server. (4) expansion. Ward's environment has become more complex, interfering factors, and each ward environment: vary in size, therefore, to ensure that senses that the terminal equipment can be used in different environments, and has a higher scalability. Intelligent Hospital care system including: electronic thermometer with a wireless communication function, pulse, blood pressure measuring instrument two parameters; wireless gateway; mobile nursing station. Through the hands of the patient physiological signal acquisition device and the wireless gateway deployed in the ward corridor, the transmission of physiological parameter information to the hospital information system, ward physiological parameter intelligent acquisition.

R \& D focus on technology: the use of 433MHz communications bands NRF905 low-power RF radio frequency communication module, combined with low power MCU and physiological signal sensing device to the general ward daily blood pressure must be measured: body temperature and pulse three key parameters acquisition, analysis and processing. Accordingly, in the perception layer developed a wireless electronic thermometer and wireless blood pressure and pulse count. RF radio frequency communications networks into a Wifi communication, and enhances the functionality and automatic temporary storage networking. In making the application layer control system PC real-time acquisition of physiological signals based on critical care system and PDA based.

\section{The Sensor Layer Design}

Temperature sensor consists of temperature measurement circuit, the main chip, wireless transmission module, power management module. Temperature measurement circuit mainly by JA31104 chip and composition, the main chip selects TI's chips, wireless transmission module selection NRF905 module. Temperature measurement chip temperature into an electrical signal, the master chip reads the electrical signal by interrupting the way, and calculate pulse data OEB pin JA31104 chip to achieve read temperature data. Master chip count by interrupting the way to judge whether the temperature is the maximum temperature, the highest temperature of its preservation. When the master chip stable maximum temperature starts to configure the wireless transmission module, reduce system power consumption and extend battery life.

Key design considerations shall be a body temperature sensor when the following points: (1) must meet the requirements of medical temperature measurement, measurement error is 0.1 or less; (2) simple operation, anyone can quickly use the thermometer; (3) the housing seal surface smooth, has antibacterial and anti-sterilization function; transmission and control (4) data without human involvement and interference; (5) designs measurement error compensation function to solve the thermistor temperature drift caused by aging; (6) low power consumption and extend battery life.

The blood pressure monitor uses high-performance sound pressure sensor, by extracting auscultation of korotkoff sound signal determining blood pressure values. The method can eliminate oscillography electronic sphygmomanometer individual differences, raise blood pressure measurement accuracy. The design process developed korotkoff latency technologies to further accurately determine the systolic and diastolic blood pressure, ensure the accuracy of the individual measurements. Using high-precision pressure sensors and custom korotkoff sound sensor, a blood pressure-related signal extracted by linear deflation and pressure auto-zero technology, improve overall system performance. Mercury manometer liquid crystal simulated appearance, scale, high resolution, can accurately display the cuff pressure, the cuff can be observed pulsating signal. Full information is recorded and got more information about the status of the body other than the arterial blood pressure. 


\section{The Network Layer Design}

Physiological signal gateway is a program that automatically converts the RF signal to the device Wifi signal, taking into account the different wards physiological signals would send a design crosstalk, physiological signal gateway must meet today's complex application environments. Things gateway is different from the current Internet gateway, the gateway not only to complete the things the Internet gateway data forwarding function, but also to achieve the transformation of different communication frequency bands, the timing for different network environments. Project Design physiological parameters need to customize the gateway networking functionality and dynamic configurable functions. By ward environment vary widely, from networking features and dynamically configurable function guarantees no signal place simply by adding a wireless gateway will be able to work. Ward physiological parameter monitoring transmission basically occurs at the same time, most of the hospitals prescribed three times daily temperature measurement, so the possibility of more beds in local data concurrency is very large, the RF communication system on the use of carrier sense and collision avoidance way to solve this problem.

\section{The Application Layer Service Software Design}

The main function of the service application layer software is to maintain the life of parameter information, display, acquisition and write to view and alarm mainly for maintenance of the database maintenance functions, including add, delete and updated records. The man-machine interface based on $\mathrm{C}++$ languages, development tools, application-based $\mathrm{VC}++6.0 \mathrm{MFC}$ dialog class. the basic process is the transfer of patients from the hospital HIS in the basic information display area of the disease, including patient ID bed number, room number, diagnostic information, Physician hygiene, nurses and doctors can visually see the patient information, life parameters transferred to a PC or server, can be displayed in real time on the screen, after receiving the doctor or nurse complete click the save button to save the parameter data to the server. The reception data show that in the corresponding column of the patient, but also saved when you save the information according to the patient, and the patient vital signs parameters dislocation will not happen. You can also modify the perception HMI terminal equipment number, when the patient's device when damaged, the patient may be time for the replacement of equipment.

\section{Conclusion}

This paper designs and implements intelligent hospital care system based on Internet of things. In the hospital ward to improve the management level of automation, reduce staff workloads, reduce input costs, reduce patient accompany bed design principles, communication technology, photoelectric detection technology, computer technology, computer network technology and relational database technology successfully applied based things ward intelligent monitoring system, using the system to care nurses provide convenience to the daily treatment of a patient to provide a reliable security guarantee, it has a high application value.

\section{References}

[1] Huifang Zhou: Medical Equipment, Vol. 6 (2014) No 53, p.25-26

[2] Hongli Zhang: Chinese Journal of Biomedical Engineering, Vol. 12 (2015) No 27, p.74-76

[3] Qin Guo: Application Research of Computers, Vol. 1 (2016) No 33, p.11-14

[4] Jieming Liu: China Medical Equipment, Vol. 3 (2011) No33, p.121-124

[5] Han Ming: Intelligent Computer and Applications, Vol. 3 (2011) No33, p.121-124 